\title{
Consensus conference on Lyme disease
}

Os

N JANUARY 15-16, 1991. A CONSENSUS CONFERENCE on Lyme disease, cosponsored by the Laboratory Centre for Disease Control, Health and Welfare Canada, and the Canadian Infectious Diseases Society, was held at the University of Guelph in Guelph, Ontario. The purpose of this conference was to achieve a consensus on Lyme disease issues and to issue statements in four areas:

\section{Epizootiology \\ II. Epidemiology \\ III. Clinical practice \\ IV. Laboratory investigation}

Participants included representatives from universities and governments across Canada, and specialists from the United States (where the incidence of Lyme disease is much higher than in Canada) who presented background papers reflecting their experience and expertise in each of the four areas.

Statements emerging from workshop discussions reflect a consensus based on current knowledge and are subject to change in the light of ongoing study and research. These statements are intended to serve as a guide for all those involved in the investigation, control and treatment of Lyme disease in Canada.

\section{EPIZOOTIOLOGY: Consensus statement Preamble}

Lyme disease is a tick-borne infection caused by Borrelia burgdorferi and transmitted primarily by members of the Ixodes ricinus group of ticks. In North America this group is represented by Ixodes dammini, Ixodes pacificus and Ixodes scapularis. I dammini and I pacificus are known to occur in
Canada. On the basis of current knowledge, the only area in Canada with a documented breeding population of I dammini is Long Point, Lake Erie, Ontario. There are, however, reports of I dammini elsewhere in Canada.

\section{A. Criteria for defining the status of I dammini in an area*}

The status of I dammini in an area is defined as 'established', 'adventitious', or 'not present' according to the following criteria:

1. 'Established' (endemic)

All three stages - larva, nymph and adult - are present in a locality (a contiguous sampling area) on resident animals or in the environment for at least two consecutive years.

\section{2. 'Adventitious'}

Findings are sporadic, both temporally and spatially, and usually involve a single stage of the tick.

\section{3. 'Not Present'}

Ticks are not found after the following studies:

a. Examination, in one locality, of a minimum of 30 small mammals for immature ticks under magnification or by digestion of skins in potassium hydroxide, at a time of year when immature stages are expected to be present (usually May to August). This gives a 95\% probability of detecting infestation at a prevalence of 5 to $10 \%$.

b. Close examination of the head, neck and fore-

*Adaptations of these approaches appropriate to the biology of I pacificus should be applied to establish its status in a locality. Further research is necessary to establish appropriate approaches to defining the status of I pacificus in the Canadian environment. 
quarters of a large enough sample of deer, per hunting or wildlife area, to give a 95\% probability of detecting adult ticks at a $5 \%$ prevalence (eg, 45 to 60 deer if the population in the unit ranges from 100 to 10,000 deer), at an appropriate time of year (usually October 1 to December 15).

c. Sampling of the environment for questing ticks using a $1 \mathrm{~m}^{2}$ flannel drag or flag, at the appropriate time of year (usually May to August for immature ticks, October to December 1 for adults). This should be done during favorable weather, on at least three separate days per locality, for a minimum total of 10 personhours.

On the basis of these definitions, the population of ticks on Long Point, Lake Erie, Ontario is classified as established. All other recorded findings of I dammini are classified as adventitious.

\section{B. Methods of detecting B burgdorferi in tick vectors and wildlife reservoirs}

\section{In regions where I dammini is endemic}

Attempt to demonstrate $B$ burgdorferi in ticks by examining up to 100 individual nymph or adult ticks by darkfield or immunofluorescent antibody staining (IFA) for B burgdorferi. If spirochetes are recognized, attempt isolation and identification of $B$ burgdorferi

a. in 100 nymphs or adults (in a minimum of 10 pools of 10 ticks)

\section{OR}

b. in 30 individual small mammals by culture of ears and bladders.

While serological studies in animal hosts may assist in raising the index of suspicion for endemic $B$ burgdorferi, serology must be interpreted with caution; it must not be used to define an area as endemic or nonendemic for B burgdorferi.

\section{In regions where I pacificus is endemic}

Demonstrate $B$ burgdorferi in I pacificus by darkfield microscopy or IFA as a screening test, saving the positive ticks for culture. Culture surveys of small mammals are not appropriate in areas endemic for I pacificus, since the prevalence of infection is expected to be very low.

3. In regions where neither I dammini nor

\section{I pacificus are endemic}

Expert opinion, based on current knowledge, is that it is not reasonable or necessary to search for $B$ burgdorferi in the environment. The wood tick or American dog tick, Dermacentor variabilis, is not a competent vector for Lyme disease. Further research into the role of other alternative vectors is desirable.

\section{General recommendations regarding epizootic surveillance}

1. The approaches to establishment of the endemic status of I pacificus, I dammini and $B$ burgdorferi described in A and B above, if negative, are sufficient to conclude with reasonable confidence that the Lyme disease cycle is not present in nature in a locality.

2. Passive surveillance for I dammini and I pacificus on people and wild and domestic animals, and examination of deer for $I$ dammini and $I$ pacificus, are encouraged. Field investigations for endemic ticks should be guided by knowledge of the biology and ecology of tick vectors, and discovery of I dammini and I pacificus, or clusters of human Lyme disease cases.

3. Epidemiological surveys for Lyme disease tick vectors and wildlife reservoirs should be supported.

4. A national reporting system for I dammini and $I$ pacificus distribution should be established.

5. The definition of endemic Lyme disease in the human population must be based on findings supporting the presence of $B$ burgdorferi and an efficient vector in the environment (if possible).

\section{EPIDEMIOLOGY: Consensus statement Preamble}

Lyme disease is generally rare in Canada. Only 140 cases were reported between 1984 and 1990 . Since case definitions were not nationally standardized and often imprecise, many of these reported cases would probably not meet the new surveillance case definitions presented below. Most Canadian cases $(71 \%$ or 100 cases) were reported from Ontario. Of the 40 cases from the rest of Canada, 17 were from Manitoba, 11 from British Columbia, five each from New Brunswick and Quebec, and one each from Newfoundland and Saskatchewan. Forty-two per cent of the Ontario cases and $97.5 \%$ of cases reported from the other provinces had a history of travel to a recognized endemic area outside of the reporting province during the presumed incubation period.

Use of the new case definitions to re-evaluate existing cases and to assess cases reported from ongoing surveillance will be instrumental in obtaining a more accurate estimate of the frequency of Lyme disease in Canada. Mandatory reporting of Lyme disease is recommended in provinces or territories where there is evidence of endemic foci. Voluntary reporting of cases is encouraged in all other areas. 


\section{A. Methods for monitoring trends where the apparent incidence of Lyme disease is low}

The trigger for both human and epizootiological studies is the occurrence of human cases or vector ticks that may be made known through passive surveillance methods.

\section{Human studies}

a. Standardized collection of data on individual cases provides valuable epidemiological information;

b. Surveillance of hospital discharge diagnoses identifies cases of Lyme disease that have not been reported;

c. Study of hospital discharge databases (HMRI, Med-Echo) helps to assess trends in the occurrence of hospitalization for Lyme disease;

d. Serosurveys are generally believed to be of very limited value.

\section{Epizootiological studies}

Epizootiological studies monitor trends in the distribution of I dammini and other potential vectors (see I. Epizootiology: Consensus statement).

\section{B. Case definitions for Canadian national surveillance of Lyme disease ${ }^{\circ}$}

The following are surveillance definitions and are not intended to guide clinical management. Failure to meet a surveillance case definition does not preclude treatment which, instead, should be initiated on the basis of clinical judgement. Since the epidemiology of Lyme disease is not fully known, and since laboratory methodology is evolving, the case definitions will require review as experience is gained with the disease.

\section{Confirmed Case}

One of the following:

1. Isolation of $B$ burgdorferi from tissue or body fluid by a laboratory of demonstrated competence.

2. History of exposure in an endemic area ${ }^{1}$ and either of the following:

a. erythema migrans ${ }^{2}$ observed by a physician;

b. at least one clinically compatible late manifestation $^{3}$ and laboratory evidence of $B$ burgdorferi infection ${ }^{4}$

3. No history of exposure in an endemic area ${ }^{1}$ and both of the following:

a. erythema migrans ${ }^{2}$ observed by a physician;

b. laboratory evidence of $B$ burgdorferi infection ${ }^{4}$.

\footnotetext{
-Developed by the Advisory Committee on Epidemiology in collaboration with the Technical Advisory Committee.

-For clinical manifestations and guidelines for the treatment of Lyme disease, refer to III. Clinical Practice: Consensus statement.
}

\section{Probable Case}

One of the following:

1. History of exposure in an endemic area ${ }^{1}$ and physician recognition of erythema migrans ${ }^{2}$ as reported by the patient.

2. No history of exposure in an endemic area ${ }^{1}$ and both of the following:

a. at least one clinically compatible late manifestation ${ }^{3}$;

b. laboratory evidence of $B$ burgdorferi infection ${ }^{4}$.

\section{Notes}

1. Exposure in an endemic area

Living in, or visiting, an endemic area ${ }^{\mathrm{a}}$. Such exposure should have occurred no more than 30 days prior to onset of erythema migrans or no more than one year before the onset of late manifestations. A history of tick bite is NOT required.

\section{a. Endemic area}

One in which the risk of transmission of Lyme disease to humans is supported by either of the following:

i. the presence of an established vector population known to be infected with $B$ burgdorferi; ii. the occurrence of at least three confirmed human cases, with adequate histories, for whom there are no histories of exposure in previously identified endemic areas (a provisional definition of an endemic area in the absence of appropriate tick studies).

There is no time limit within which cases must occur or infected vectors be identified for an area to be declared endemic. The geographic limits of the endemic area will be defined by the provincial or territorial health authorities.

\section{Erythema migrans}

An erythematous expanding lesion, at least 5 $\mathrm{cm}$ in diameter, with central clearing. The lesion occurs within 30 days of exposure. Annular erythematous lesions occurring within $48 \mathrm{~h}$ of a tick bite may represent hypersensitivity reactions and do not qualify as erythema migrans.

\section{Late manifestations}

These include any of the following, when all other known causes have been ruled out:

a. Musculoskeletal system

Recurrent, brief attacks (lasting weeks or months) of physician-observed large joint swelling in one or a few joints, or chronic progressive arthritis preceded by brief attacks. Chronic progressive arthritis NOT preceded by brief attacks, chronic symmetric polyarthritis, arthralgias, myalgias or fibromyalgia syndromes are NOT accepted as criteria for musculoskeletal involvement. 


\section{b. Nervous system}

Lymphocytic meningitis, cranial neuritis, facial palsy, radiculoneuropathy, or rarely, encephalomyelitis. Headache, fatigue, paresthesias or stiff neck are NOT accepted as criteria for neurological involvement.

\section{c. Cardiovascular system}

Acute onset atrioventricular conduction defects that resolve in days to weeks. Palpitations, bradycardia, bundle branch block or myocarditis are NOT accepted as criteria for cardiovascular involvement.

4. Laboratory evidence of $\boldsymbol{B}$ burgdorferi infection Any one of the following findings, determined in a laboratory of demonstrated competence, provides laboratory evidence of $B$ burgdorferi infection:

a. Immunospecific staining of the spirochete in tissue or body fluid;

b. Significant changes in confirmed antibody response to $B$ burgdorferi in sequential serum samples;

c. Serum positive by enzyme-linked immunosorb ent assay (ELISA) serology according to recognized cutoff values and also positive by Western blot.

\section{Comparison of case definitions in Canada and the United States}

A Canadian confirmed case would meet the current United States confirmed case definition. A United States confirmed case would meet either the Canadian confirmed or probable case definition, depending on whether there was exposure in an endemic area.

1. The reasons for differentiating endemic and nonendemic areas are:

a. to obtain more accurate incidence figures for low incidence areas (most of Canada) by reducing the number of false positives among those who meet the case definition;

b. to provide a focus for prevention activities;

c. to identify areas for epizootiological study.

2. The incidence of Lyme disease in the United States is so much higher than in Canada that slight differences in the case definitions are unlikely to affect comparisons of incidence in the two countries.

\section{Needs and approaches for disseminating accurate information on Lyme disease}

In local public health jurisdictions, a central person or agency should provide all information to health care workers and the public (including the media). Information should be accurate, frequently updated, and provided in different formats.

\section{Health care workers}

a. The medical community needs information on the diagnosis and management of patients with Lyme disease, including interpretation of laboratory results, treatment guidelines and case reporting.

b. Public health staff need guidelines for investigation and follow-up of reported cases.

c. Veterinarians need information about such factors as the incidence of Lyme disease and the presence of vectors.

These groups can be approached through professional organizations, newsletters and continuing education programs.

\section{The public}

a. The public needs accurate information regarding the incidence of Lyme disease, the presence of vectors, prevention of tick bites, signs and symptoms of Lyme disease, the availability of treatment and prognosis.

Approaches to the public include media releases, fact sheets, brochures, posters and community meetings. Involvement with advocacy groups may be useful if there is a constructive relationship that permits the sharing of resources and information.

\section{CLINICAL PRACTICE: Consensus statement Preamble}

Lyme disease is an illness with dermatological, neurological, cardiac and/or rheumatological features. Patients may present with localized skin involvement (erythema migrans), or with disseminated acute or chronic disease. Most patients do not know whether they have been bitten by a tick before onset of symptoms. Patients who do not meet the surveillance case definitions (see II. Epidemiology: Consensus statement, B) may be eligible to receive treatment based on the clinical manifestations described below.

\section{A. Clinical manifestations suggestive of Lyme disease}

\section{Localized disease}

\section{a. Dermatological}

Erythema migrans may occur three to 30 days after a tick bite. Erythema migrans is an expanding, annular, erythematous skin lesion that is characteristically at least $5 \mathrm{~cm}$ in diameter and usually not pruritic or tender. Approximately two-thirds of patients with Lyme disease will have erythema migrans at some time during the acute phase of their illness. Erythema migrans should be differentiated 
from fixed drug eruptions and erythema annulare. Erythema migrans is sufficient for diagnosis of Lyme disease. True erythema migrans lesions less than $2.5 \mathrm{~cm}$ in diameter will increase in size if observed over 24 to $48 \mathrm{~h}$. Since erythema migrans is an early manifestation of Lyme disease, serological testing may be positive in only 20\% of patients. Biopsy of erythema migrans lesions is encouraged, because it may help confirm the disease when specific stains are used.

\section{Disseminated disease}

\section{a. Dermatological}

Multiple erythema migrans lesions indicate disseminated disease, and any associated neurological symptoms, including headache, should be carefully evaluated.

\section{b. Neurological}

Fifteen to $20 \%$ of patients may have neurological signs and can present with the disseminated acute and chronic forms of disease. Bell's palsy, lymphocytic meningitis, other cranial or radiculoneuropathies, or meningoencephalitis may occur, but are very unlikely to be the only manifestations of Lyme disease in nonendemic areas. Cerebrospinal fluid should be examined in patients with neurological signs or symptoms.

\section{c. Cardiac}

Cardiac disease occurs in 4 to $8 \%$ of cases of Lyme disease. Acute onset of first, second or third degree atrioventricular conduction defects may occur with the disseminated acute form of disease, but these conditions are usually transient. Isolated conduction defects are unlikely to be due to Lyme disease in patients who have not been in endemic areas.

\section{d. Rheumatological}

Arthritic involvement is characterized by recurrent, brief attacks (lasting weeks or months) of large joint swelling in one or a few joints. Uncommonly, chronic progressive (erosive) arthritis of a large joint may follow brief attacks. Chronic progressive arthritis not preceded by brief attacks, chronic symmetric polyarthritis, arthralgias, myalgias or fibromyalgia syndromes are not accepted as criteria for musculoskeletal involvement.

\section{B. Indications for specific serological testing for Lyme disease}

1. Serological testing is indicated in patients with erythema migrans, and in patients with signs of disseminated Lyme disease who have a his- tory of erythema migrans or exposure in an endemic area.

2. Obtaining sequential sera from patients with erythema migrans in areas not known to have Lyme disease can be of diagnostic value and can help to define the geographic distribution of disease.

3. Chronic fatigue and fibromyalgia without objective signs of Lyme disease are not considered manifestations of Lyme disease, and should not prompt serological testing.

4. Appropriate quantitative cerebrospinal fluid antibody levels may be useful for patients with possible neurological disease; however, such tests are currently not available in Canada.

\section{Interpretation of positive Lyme serology in patients}

1. Interpretation of serological tests for Lyme disease using currently available methods requires consideration of the signs and symptoms of the patient and whether the patient has been in an area known to have Lyme disease. In nonendemic areas, most positive tests will be falsely positive unless there is a very high clinical suspicion of Lyme disease. A positive test will confirm illness in a patient with erythema migrans or a history of erythema migrans and other typical manifestations of the disease.

2. A positive serological test in an asymptomatic patient is of no diagnostic value. Positive serological tests in a patient with atypical symptoms do not necessarily imply evidence of the disease.

\section{Guidelines for treatment}

1. Erythema migrans; Bell's palsy without cerebrospinal fluid abnormalities; first degree heart block

Adults: Oral doxycycline $100 \mathrm{mg}$ bid for two to three weeks or oral amoxicillin $500 \mathrm{mg}$ tid for two to three weeks plus oral probenecid $500 \mathrm{mg}$ tid for two to three weeks.

Children: Oral amoxicillin $40 \mathrm{mg} / \mathrm{kg} /$ day in three doses for two to three weeks plus oral probenecid $40 \mathrm{mg} / \mathrm{kg} /$ day in three doses for two to three weeks.

Doxycycline should not be used in pregnancy or for children less than nine years old. Erythromycin can be used but has been found to be less effective clinically.

2. Central nervous system disease with cerebrospinal fluid abnormalities; high degree (second or third) atrioventricular block; arthritis 
Adults: Intravenous ceftriaxone $2 \mathrm{~g} /$ day once daily for two weeks

Children: Intravenous or intramuscular ceftriaxone $100 \mathrm{mg} / \mathrm{kg} /$ day every $12 \mathrm{~h}$ for two weeks

\section{E. Treatment outcomes}

1. Treatment is 95 to $100 \%$ effective for erythema migrans, 90 to $95 \%$ effective for other acute manifestations, and 80 to $85 \%$ effective for chronic disease.

2. Herxheimer reactions occur in 15 to $30 \%$ but are usually mild and can be treated with nonsteroidal anti-inflammatory drugs.

3. Post infectious fatigue syndromes may occur in approximately $15 \%$ of treated Lyme disease patients but usually resolve spontaneously in six to 12 months.

4. In the absence of objective signs of Lyme disease, treatment is rarely useful. In these situations, the potential risks associated with treatment may outweigh any potential benefits. The most frequent reason for treatment failure is misdiagnosis; other diagnoses should be considered in such situations.

\section{LABORATORY INVESTIGATION: Consensus statement \\ Preamble}

Definitive laboratory diagnosis of human Lyme disease depends upon the detection of $B$ burgdorferi or adequate serological evidence of infection. Current serological tests lack sensitivity and specificity. These tests also show a high degree of variability. For these reasons, an effective reference service is essential. In light of the above, serological tests are indicated only for individuals who have travelled to endemic areas and/or have signs and symptoms compatible with Lyme disease. Moreover, serological studies may be negative early in the disease. Antibody formation may be aborted by early treatment. Alternatively, antibodies may persist after adequate treatment. Appropriate use of serological diagnostic studies for highly suspect cases will minimize misinterpretation of results (see III. Clinical Practice: Consensus statement). It is anticipated that the limitations of current methodologies will be overcome by new and emerging diagnostic methods that will greatly improve the reliability of serological testing.

\section{A. Recommendations for standardization and use of laboratory tests for diagnosis of Lyme disease}

1. All laboratories in Canada should use ELISA kits (from a commercially available source) for initial testing of both IgG and IgM antibodies to $B$ burgdorferi.

2. Additional diagnostic tests, such as IFA tests may be used as an adjunct to ELISA.

3. Serological results should be reported as positive, negative or indeterminate, according to defined laboratory criteria. They should not be reported quantitatively.

4. Significant changes in confirmed antibody response in sequential serum samples are considered to be diagnostic of recent infection.

5. When neuroborreliosis is suspected, routine biochemical and cytological cerebrospinal fluid examination is indicated. Reliable specific antibody testing for cerebrospinal fluid is not generally available at present.

6. Western blot should be used to confirm the presence of specific antibodies in sera found positive in the initial ELISA test. Some false positive results may be ruled out by Western blot. Standards for interpretation of Western blot must be developed.

7. A central reference laboratory should be adequately supported to undertake proficiency tests, establish standards for reference reagents, and evaluate commercial products.

\section{B. Recommendation for the use of low yield laboratory procedures to confirm the presence of Lyme disease in Canada}

Isolation of $B$ burgdorferi (from biopsies at the advancing edge of erythema migrans lesions) or identification by immunospecific staining is encouraged when possible.

\section{Recommendations for the use of newer or less common methods of laboratory diagnosis}

1. At this time, methods for laboratory diagnosis of Lyme disease such as antigen detection by ELISA, lymphocyte transformation and detection of circulating immune complexes are not appropriate for routine clinical diagnosis.

2. Development of the polymerase chain reaction and antibody detection assays based on synthetic peptide and recombinant proteins for Lyme disease diagnosis is encouraged. 


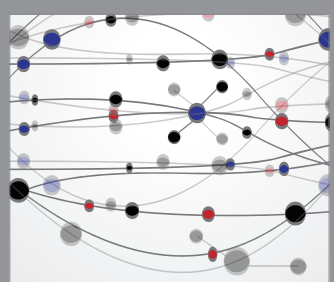

The Scientific World Journal
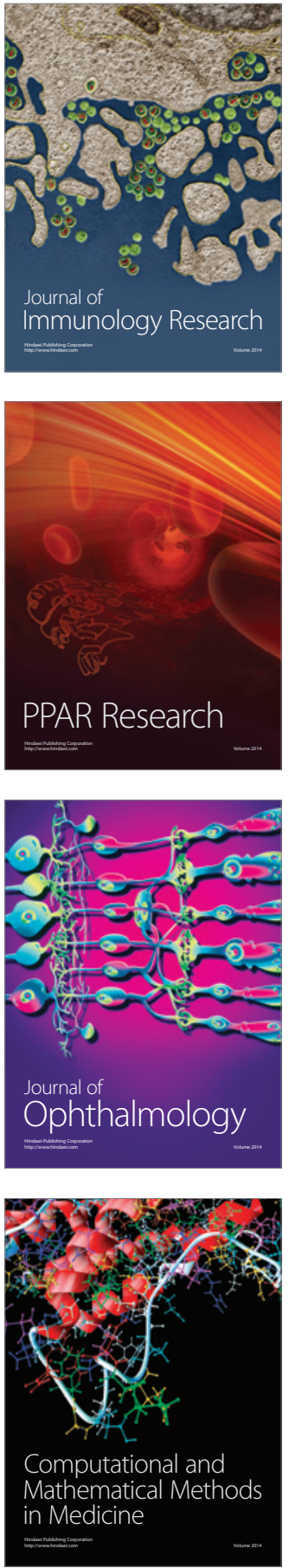

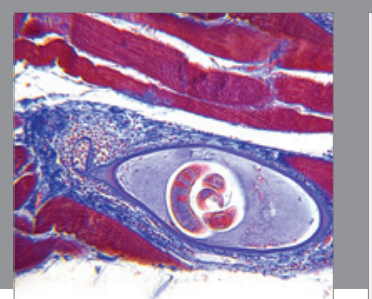

Gastroenterology Research and Practice

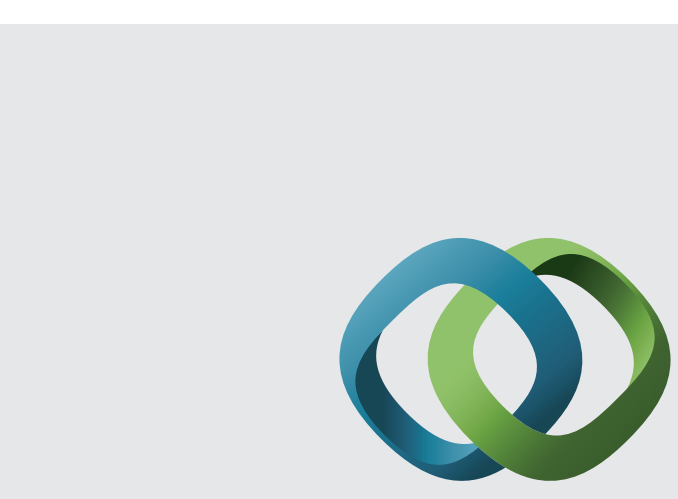

\section{Hindawi}

Submit your manuscripts at

http://www.hindawi.com
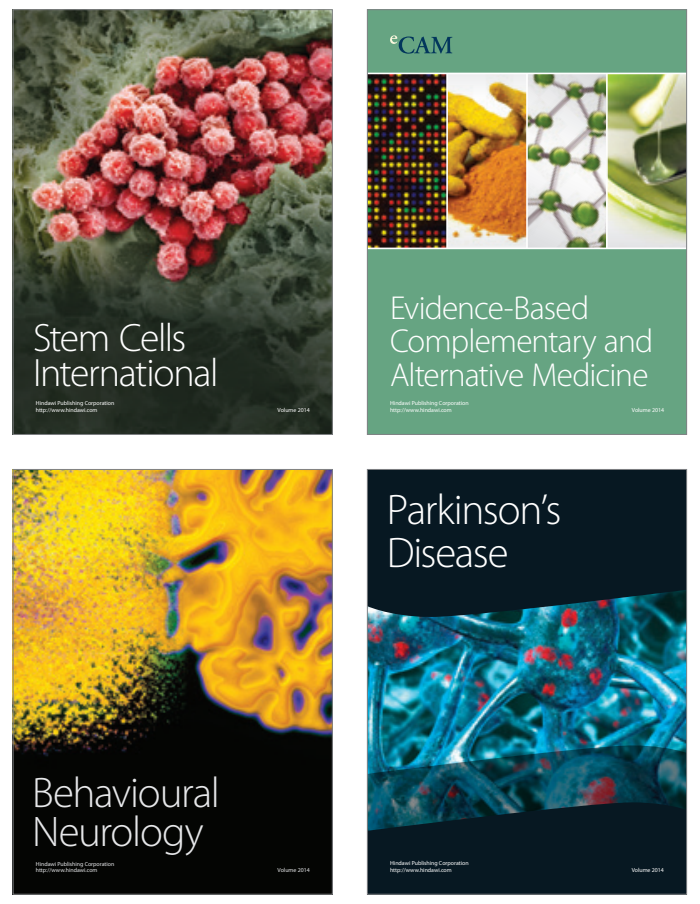
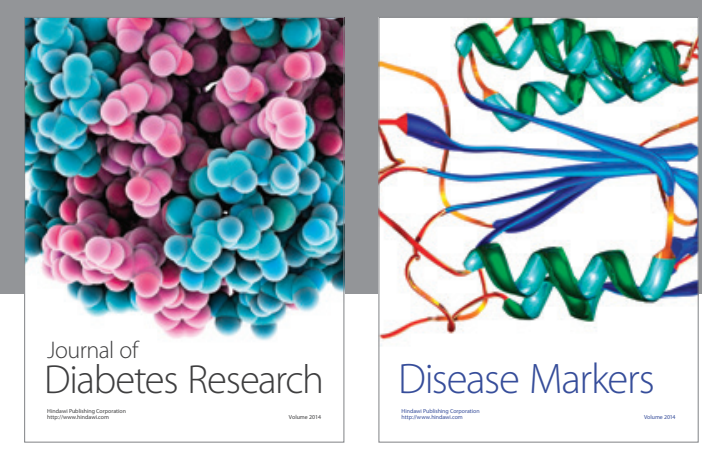

Disease Markers
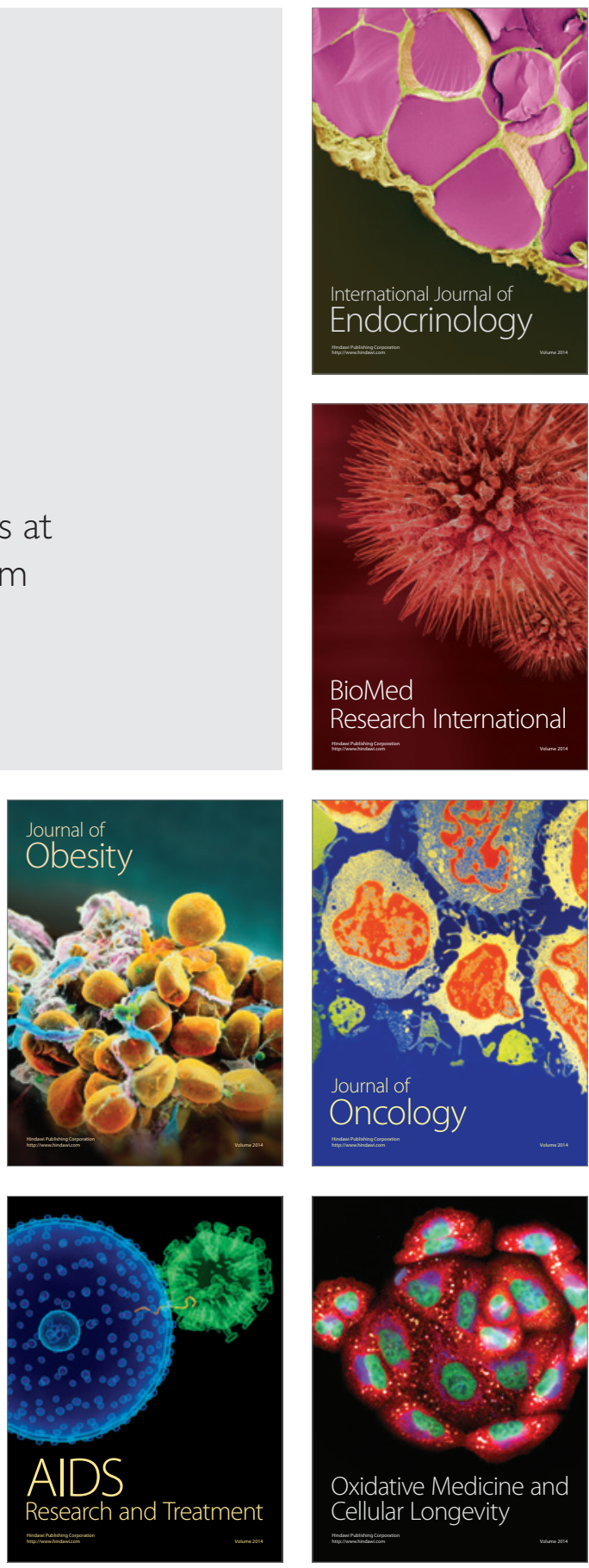